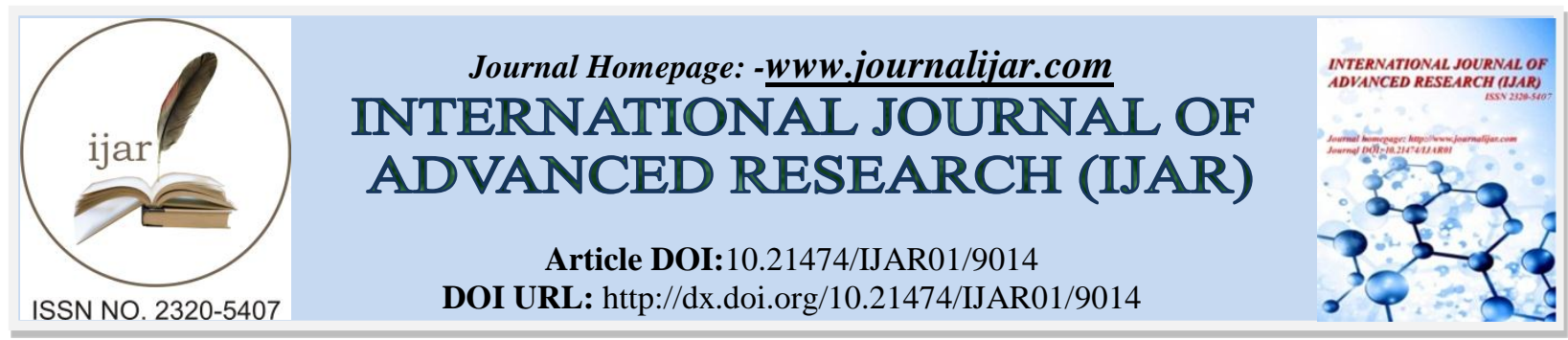

RESEARCH ARTICLE

\title{
COMPARATIVE STUDY OF RELATIONSHIP BETWEEN MEMORY STRENGTH, DEMOGRAPHIC VARIABLES AND SLEEP TIMING AMONG NORMAL ADULT PEOPLE: A CASE-CONTROL STUDY IN IRBID GOVERNORATE / JORDAN.
}

Dr. Eham Saleh Al-Ajlouni.

Jordan Ministry of Health - Amman.

\section{Manuscript Info}

Manuscript History

Received: 02 March 2019

Final Accepted: 04 April 2019

Published: May 2019

Key words:-

daytime, memory loss , memory

strength, night, Sleeping.

\begin{abstract}
Most of researches studied the effects of sleep deprivation or sleep disorders. Others studied the mechanism of sleeping function. In this study, the participants were normal adult people who had different time of sleeping; so the main objective of this study was studying the effect of sleep timing on memory strength. Whereas the specific objectives were assessment of the strength of memory of participants, comparing the memory scores between those who sleep at daytime and those who sleep at night, and comparison of memory strength among the two groups according to demographic variables. It was found that majority of cases sleep after the Dawn while majority of controls sleep after midnight. Also, the average scores of each of all ten questions of the cases were lower than of the controls. Moreover, the average of total scores of all questions of memory test among the cases were lower than among the controls. However, the differences were not significant. But when the scores categorized into low, medium and high, the differences became significant at alpha level 5\%. Lastly, it was concluded that there is not significant association between time sleeping and high memory strength; but there is significant association between time of sleeping and low memory strength. Furthermore, there is no effect of level of education and income on memory strength; sex and marital status has effect on memory of night people (i.e. cases); while age group has effect on memory of control group.
\end{abstract}

Copy Right, IJAR, 2019,. All rights reserved.

\section{Introduction:-}

\section{Rationale and importance}

Sleep makes human being feel better, but its importance goes beyond just promoting the mood or casting out bags under eyes. Adequate sleep is a significant part of a healthy lifestyle, and can affect vital organs like heart. Also, may affect mind, weight and more. (Mograss et al, 2008) Epidemiologic studies have found that sleep duration is associated with obesity, diabetes, hypertension and mortality. These studies have used self-reported habitual sleep duration. (Lauderdale et al, 2008) Also individual differences in sleep in healthy adults have been investigated. Several electrophysiological studies concerned with individual differences in sleep duration reported differences in sleep structure and variables of sleep/wake. The shorter sleep hibernation and the more rapid accumulation of Slow

Corresponding Author:-Dr. Eham Saleh Al-Ajlouni.

Address:-Jordan Ministry of Health - Amman. 
Wave Sleep in the short sleepers which was explained as a consequence of higher sleep pressure at the beginning of the night. (Mograss et al, 2008)

The sleep cycle consists of two recurring phases: rapid eye movement (REM) and non-rapid eye movement (NREM). 20-25\% of total sleep each night for REM sleep. In this phase, dreaming occurs, which is important for consolidating emotions, memories and stress. It is also important for learning, arousing the brain regions used in learning and developing new skills. On the other hand, NREM sleep occupies 75-80\% of total sleep per night. In this phase, tissue repair and growth occurs, energy is restored and hormones are released. (Morselli L et al., 2010) Enhancement in performance on analytical memory tasks has been associated with early Non-REM sleep, consisting of the highest percentage of slow-wave sleep. The long sleepers, failed to show significant recall of the old/studied items, with their identification of old faces not different from chance. Correlation Coefficient revealed a significant negative correlation between accuracy and sleep duration in the short sleepers. While long and average sleepers showed a positive correlation between the two variables. (Mograss et al, 2008) If the REM and NREM cycles are interrupted many times all over the night - because of snoring, difficulties breathing or waking up frequently then vital body processes will be failed, which can affect health and well-being the next day and long term. (Morselli L et al., 2010) The National Sleep Foundation recommended that, for healthy individuals with normal sleep, 8 to 10 hours for teenagers was considered appropriate, 7 to 9 hours for young adults and adults, and 7 to 8 hours of sleep for old people. However, sufficient sleep duration vary from person to person. (Hirshkowitz et al, 2015)

One of the important parameters involved in how to sleep well is the time of going to sleep. A scientific theory said that from sunrise to sunset clockwise or positive frequencies are moving in the atmosphere. After sunset the anticlockwise or distressing frequencies start moving in the atmosphere. (Gupta, 2015)

In this study, effect of the time of going to sleep on the memory strength was invistigated, among adult normal persons.

\section{Objectives}

Therefore, the main objective of this study was studying the effect of sleep timing on memory strength. Whereas the specific objectives were assessment of the strength of memory of participants, and comparing the memory scores between those who sleep at daytime and those who sleep at night; and comparison of memory strength among the two groups with respect to demographic variables.

\section{Methodology:- \\ Eligibility}

Eligible persons for this study were people who live in Irbid governorate, and aged 27-53 years old. Case-control method was used, in which the cases were those who sleep at daytime, from Dawn to sunset. While the control group were those who sleep at night, from sunset to dawn.

\section{Memory scale}

10-item Rasch modeled memory self-efficacy scale was used to assess the memory strength. The original scale is in English, therefore it was translated into Arabic language. To assure credibility of the translated scale, three $\mathrm{PhD}$ holders in translation reviewed it. Then 12 volunteers with very good English proficiency answered both versions of the scale. To assess reliability of the translated scale, Cronbach's alpha was calculated and found to be 0.869 .

\section{Pilot study}

To check the questions, the options of questions, clarity of language, simplicity of ideas, and any confusion to participants, the questionnaire was tested twice by 42 persons ( 24 females, 20 males).

\section{Data collection}

To apply the study and collect the data, both self-administrated and reviewer-administrated questionnaires were used, over June - September 2018. In addition to online questionnaire.

\section{Results:-}

The sample of the study was consisted of 104 cases and 146 controls. However 6 cases were excluded : 3 for honesty issue, 1 case was suffering from Vitamin D deficiency, and the rest 2 cases had contradiction answers. For 
control group, 24 were removed due to unreliable data collectors. Regarding sex, $40.37 \%$ of the sample were males, while $59.63 \%$ were females. Percent of females among cases was slightly higher than among controls, $60.2 \%$ and $59.17 \%$ respectively. However, at $95 \%$ confidence level, the difference was not significant. The chi-square statistic was 0.0241 and the p-value was 0.876585 .

Regarding the age, cases were younger than controls: $50 \%$ of the cases were less than 31 years old, while $30 \%$ of controls were 41-50 followed by $29.17 \%$ were less than 31 years old. The chi-square statistic was 9.311 , and the pvalue was 0.025429 . Therefore, with a $95 \%$ confidence level, the difference between ages of cases and controls was significant.

With respect to education, majority of cases and controls were higher than secondary level of education. $70.41 \%$ of cases had college or university education compared to $62.5 \%$ of controls. On the other hand, $10 \%$ of the controls were illiterate or had elementary education compared to $6.12 \%$ of cases. The chi-square statistic was 1.8342 , and the p-value was 0.399674 . So, with a $95 \%$ confidence level, the difference of education among cases and controls was not significant.

Regarding marital status, singles (i.e. bachelors) were more among cases than controls: about half of the cases $(48.98 \%)$ were single, while two-third of the controls were married. The chi-square statistic was 15.6247 , and the p-value was 0.000405 . Therefore, with a $95 \%$ confidence level, the difference of marital status between cases and controls was significant.

According to self-evaluation of economic status of the participants, it was found that among the cases, "very good / excellent" followed by "good" status were more common, 33.67\% and $31.63 \%$ respectively. On the other side, among the controls, more than half of them had "good" economic status $(58.33 \%)$. The chi-square statistic was 16.888 , and the p-value was 0.000745 . So, with a $95 \%$ confidence level, the difference of economic status among cases and controls was significant.

Table 1:-Distribution of sleep time among cases and controls, in Irbid Governorate, 2018

\begin{tabular}{|c|c|c|c|c|c|}
\hline \multirow[t]{2}{*}{ Sleeping time } & & \multicolumn{2}{|c|}{ Cases } & \multicolumn{2}{|c|}{ Control } \\
\hline & & $\mathrm{F}$ & $\%$ & $\mathrm{~F}$ & $\%$ \\
\hline Morning & (Dawn-midday) & 71 & $(72.45 \%)$ & & \\
\hline Noon & (After midday- Asr prayer) & 16 & $(16.33 \%)$ & & \\
\hline After noon & (After Asr prayer-Sunset) & 11 & $(11.22 \%)$ & & \\
\hline Early night & (After sunset-Isha prayer) & & & 6 & $(5.0 \%)$ \\
\hline Night & (After Isha prayer-midnight) & & & 26 & $(21.67 \%)$ \\
\hline After midnight & (After 12 am - Fajir prayer) & & & 88 & $(73.33 \%)$ \\
\hline Total & & 98 & $(100 \%)$ & 120 & $(100 \%)$ \\
\hline
\end{tabular}

As shown in table 1 that majority of cases (72.45\%) sleep after dawn followed by sleeping at noon (16.33\%). While majority of controls (73.33\%) sleep after midnight followed by sleeping after Isha prayer (21.67\%) - mainly after 9 p.m. -

Regarding the reasons of sleeping at daytime, $28.57 \%$ preferred to be activated at night (i.e. night persons), and $30.61 \%$ of them due to their works, while $25.51 \%$ suffered from insomnia.

Table 2:-Distribution of period of sleeping among cases and controls, in Irbid Governorate, 2018

\begin{tabular}{|l|l|l|l|l|l|l|}
\hline \multirow{2}{*}{ Average duration of Sleeping } & Cases & \multicolumn{2}{l|}{ Control } & \multicolumn{2}{l|}{ Total } \\
\cline { 2 - 7 } & $\mathrm{F}$ & $\%$ & $\mathrm{~F}$ & $\%$ & $\mathrm{~F}$ & $\%$ \\
\hline Less Than 6 Hours & 26 & $(26.53 \%)$ & 26 & $(21.67 \%)$ & 52 & $23.86 \%$ \\
\hline 6- 7 Hours & 42 & $(42.86 \%)$ & 64 & $(53.33 \%)$ & 106 & $48.62 \%$ \\
\hline More Than 7 Hours & 30 & $(30.61 \%)$ & 30 & $(25.00 \%)$ & 60 & $27.52 \%$ \\
\hline Total & 98 & $(100.0 \%)$ & 120 & $(100.0 \%)$ & 218 & $100.0 \%$ \\
\hline
\end{tabular}


As shown in table 2, the most common average hours of sleeping in both groups was "6-7 hours". Medium period of sleeping (6-7 hours) was more common among the controls than the cases, 53.33\% compared to $42.86 \%$. On the other hand, long period of sleeping (more than 7 hours) was more among the cases than the controls, $30.61 \%$ compared to $25 \%$. However, the chi-square statistic was 2.37 and the p-value was 0.305748 . Therefore, upon the statistical analysis, with a 95\% confidence level, the difference of sleeping duration between cases and controls was not significant.

Regarding family history of Alzheimer disease, the majority of the sample did not have family history of Alzheimer disease. Patients of Alzheimer disease were more among cases' families than controls' families, $18.37 \%$ and 13.33 $\%$ respectively. However, the chi-square statistic was 1.7177 and the p-value was 0.423645 . So, with a $95 \%$ confidence level, there was no significant difference between the two groups.

Relating to assessment of memory strength of the participants, it was found that the average scores of each of all ten questions of the cases were lower than of the controls (see table 3). Furthermore, the average of total scores of all questions of memory test among the cases was lower than among the controls. However, the chi-square statistic was 4.2441 and the p-value was 0.373977 . Therefore, with a 95\% confidence level, the differences were not significant.

When the scores of memory strength were categorized into low, medium and high (as in table 4), it was found that more than half of cases $(56.12 \%)$ had medium scores, while more than half of controls $(59.17 \%)$ had high scores. The chi-square statistic was 22.2015, and the p-value was 0.000015 . Based on the statistical results, the differences of memory strength categories among cases and controls were significant at alpha level $5 \%$.

Table 3:-Distribution of average scores of 10-memory test questions among cases and controls, in Irbid Governorate, 2018

\begin{tabular}{|l|l|l|}
\hline 10-memory test & Cases & Controls \\
\hline A1. Overall assessment of your memory status? & 3.82 & 4.31 \\
\hline A2-1. Problems with names? & 4.05 & 4.52 \\
\hline A2-2. Problems with faces? & 4.90 & 5.55 \\
\hline A2-3. Problems with where you put your key, ..? & 3.74 & 4.43 \\
\hline A2-4. Problems in directions? & 5.03 & 5.26 \\
\hline A2-5. Problems with remembering what are you doing & 4.35 & 5.39 \\
\hline A3-1. Remembering the paragraph before your last paragraph? & 4.13 & 4.87 \\
\hline A3-2. Remember the sentence before your last sentence? & 4.32 & 5.15 \\
\hline A4-1. Remember things before 1-5 years left? & 4.87 & 5.26 \\
\hline A4-2. Remember things before 6-10 years left? & 4.43 & 4.91 \\
\hline Total scores & $\mathbf{4 3 . 6 3}$ & $\mathbf{4 9 . 6 4}$ \\
\hline
\end{tabular}

Table 4:-Distribution of memory score categories among cases and controls, in Irbid Governorate, 2018

\begin{tabular}{|l|l|l|l|l|}
\hline \multirow{2}{*}{ Memory scores } & Cases & \multicolumn{3}{l|}{ Controls } \\
\cline { 2 - 5 } & $\mathbf{F}$ & $\mathbf{\%}$ & $\mathbf{F}$ & $\mathbf{\%}$ \\
\hline Low (total score is less than 30) & 14 & $14.29 \%$ & 4 & $3.33 \%$ \\
\hline Medium (total score is 30-50) & 55 & $56.12 \%$ & 45 & $37.5 \%$ \\
\hline High (Total score is more than 50) & 29 & $29.59 \%$ & 71 & $59.17 \%$ \\
\hline Total & 98 & $100 \%$ & 120 & $100 \%$ \\
\hline
\end{tabular}

Additionally, it was interesting to find that in comparison between high memory scores versus low / med scores, among cases and controls, it was found that Odds ratio was 1.6421 ; 95\% CI was [0.9321-2.8928] and significance level was $\mathrm{P}=0.0860$. The confidence interval crosses 1.0. This implies there is no difference between the groups; and $\mathrm{P}>0.05$ indicates that there is not a statistically significant difference. Therefore, there is not significant association between time sleeping and high memory strength. But, when the categories of memory strength were changed to low versus med/high, the calculated Odds ratio became $0.0057 ; 95 \%$ CI became [0.0018 - 0.0181; and z statistic was 8.822 at the significance level $\mathrm{P}<0.0001$. OR is $<1$ which indicates that cases is better than the control with regard to low memory strength. Also, The confidence interval did not crosses 1.0, which implies there is 
difference between the groups; and $\mathrm{P}<0.05$ indicates a statistically significant difference between groups. This implies that there is significant association between time of sleeping and low memory strength.

Furthermore, for demographic variables of cases and controls: statistical analysis revealed no significant differences among cases and control, with respect to memory strength and both level of education and economic status. Additionally, there was significant differences among categories of memory strength (low vs. med/high) of cases (pvalue $=0.016 ; 0.05$ respectively), while there was not significant differences among control group, with respect to both sex and marital status. On the other hand, there was significant difference among control ( $p$-value $=0.017$ ) but not among cases, with respect to age groups.

\section{Discussion:-}

The sample of the study consisted of 218 participants in which females were more than males, more than half of participants were 40 or less years old, which is expected since the Jordanian population is young. (Jordan Times, 2018) Most of their level of education was college or university, more than half of them was married, and many of the sample evaluated his/her economic status as good. Self-evaluation of economic status was a good method to avoid non-response or false information from some participants. Monthly income not only sensitive issue, but also does not take into account if it is enough with respect to family size and financial commitments. When comparison between cases and controls, it was found that cases were younger, more single person marital status and less financial status. Also, most cases used to go to sleep after sunrise and before midday, while most controls went to sleep after midnight and before sunrise. Additionally, there was no significant difference between them regarding number of sleeping hours and family history of Alzheimer. Moreover, it was found that half of the cases were less than 31 years old, which means young. This is consistent with the age structure of Jordan population in which $67.78 \%$ of them less than 30 years old.( DOS, 2016) Also, may be social activities and fun at night encourage them to keep wake up till late time, or maybe they seek for privacy and calmness to perform their tasks at night. The common reasons for the delayed sleep usually homework, get along with friends, late night television screening, and video games internet. (Mishra et al, 2018) The introduction of media in bedrooms like television, internet, mobile phones, and video games were largely held responsible for sleep debt. Studies have reported that watching television lately reduced not only the total sleep time, but also delayed bed time and difficulty in waking up. (Mishra et al, 2018) These explanations can be applied too on the findings of single persons (i.e. bachelors) among the cases of this study.

Female cases were more than male cases, which is consistent with rate of unemployment. $13.3 \%$ of males were unemployed, while $24.1 \%$ of females were unemployed. (DOS, 2016) this may explain why unemployed female cases sleep at daylight.

Moreover, it was found that economic status of cases was very good / excellent. This finding is controversial. The famous inventors Thomas Edison and Leonardo da Vinci both used poly-phasic sleep cycles, in which they took lots of naps throughout the day instead of sleeping at night. (Scott, 2018) Whereas Bill Gates (an American computer programmer and inventor) goes to sleep at 1a.m. and Winston Churchill (a former British prime minister) goes to sleep at 3 a.m. (Walton, 2015)

For insomnia, the prevalence was considerably high. Although it is generally believed that $10 \%$ to $15 \%$ of the adult population suffers from chronic insomnia, and an additional $25 \%$ to $35 \%$ have transient or occasional insomnia, prevalence estimates vary because of inconsistent definitions and diagnostic criteria. (Doghramji, 2006)

Regarding number of sleep hours, previous studies have shown that both too few and too many sleeping hours are linked to a number of health problems over time and even early mortality. There are about seven sleeping hours, depending on the individual, can indicate underlying problems. (Walton, 2015) In this study, it was found in both groups that the more common sleep duration was "6-7 hours", however cases sleep longer hours than control. According to the inaugural sleep survey by Tempur, back in 1942 only $14 \%$ of people averaged six hours of sleep or less per night. However, in 2013, those getting six hours of sleep or less each night had risen to $41 \%$. In fast-moving society, six or seven hours of sleep may sound pretty good. (Smith et al, 2018) Sleep requirements vary slightly from person to person, most healthy adults need between 7 to 9 hours of sleep per night to function at their best. But not only getting enough each night, but also getting good-quality sleep. (Taylor, 2017; Smith et al, 2018) There's no universal definition for sleep quality. However, it may be defined as how long it takes to fall asleep, how often wake up during the night, or how rested person feel the next day. (Taylor, 2017) There is a big difference between the amount of sleep hours and the amount need to function optimally. According to the National Institutes of Health, the 
average adult sleeps less than seven hours per night. (Smith et al, 2018) Nevertheless, It is known that both good quality sleep and adequate amount of sleep are important in order to have better cognitive performance and avoid health problems and psychiatric disorders. (Giri, 2013)

In this study, scores of "10-item Rasch modeled memory self-efficacy scale" were lower among cases for all questions, but this was not statistically significant. May be time of sleep affect the concentration but not the memory strength. In a survey of 294 college students, it was shown significant main effects of sleepiness on mood states based on the Profile of Mood States. (Jean-Louis, 1998) Also, in a study published in Springer's Cognitive Therapy and Research Journal found that "night" people who went to bed later had more negative thoughts and contemplation than "morning" people who went to bed earlier. Another study found that Japanese workers who went to bed later showed more depressive symptoms than those who went to bed earlier. Whereas a study found that "morning" people may avoid mental illness. (Faith Xue, 2019) A cross-sectional study in Rabat, Morocco was conducted on 457 medical students from the Faculty of Medicine and Pharmacy, in which they completed the Pittsburgh Sleep Quality Index (PSQI) questionnaire and the Epworth Sleepiness Scale, in order to determine the quality of sleep and excessive daytime sleepiness, respectively. It was found that daytime sleepiness was not statistically associated with academic performance. (El Hangouche, 2018) In addition, Harvard researchers found that not staying up late or sleeping in is not the problem, but irregular sleep patterns are. Irregular sleep patterns were associated with poorer performance and productivity. According to their results, person can go to sleep and wake up at any time. The important thing is to keep a steady schedule. This can explain that productivity of night shift people as nurses and security, and night work people as celebrities and artists can be high. Charles Czeisler, MD, chief of the Sleep and Circadian Disorders Division at Brigham and Women's Hospital, said "If you go to bed at 2 and get up at 9, that's fine. You just have to consistently do the same thing." (Faith Xue, 2019)

But when scores were categorized into low, medium and high. The differences between cases and control became statistically significant which is consistent with many findings of studies. Furthermore, it was interesting to find significant differences when compared low versus medium/high categories of cases and control, while no significant differences were found when compared high versus medium/low categories . The latter (i.e. high category) can be attributed to genetic predisposition factor in which some people have inherited strong memory, while the former (i.e. low category) can be attributed to hormone role related to sleeping quality and life style. It was found that Sleep plays an important role in the consolidation of memory. This has been most clearly shown in adults for both procedural memory (i.e. skills and procedures) and affirmative memory (e.g. recall of facts). (Potkin, 2012) In a cohort study, 285 children aged 6-49 months were studied over four years. It was found that Physical Activity was significantly correlated with earlier waking time, and late risers were found to be more inactive. This could be attributed to morning light. (Kohyama, 2007)) Morning light is known to be a powerful signal for shortening the circadian rhythm. Consequently, morning light harmonizes the circadian rhythm with the 24 hour cycle of the earth. (Christoph, 2009) In addition, light exposure in the morning has been reported to be effective for depressed patients and seasonal affective disorder. (Kohyama, 2007) Another study found that irregular sleep and light exposure patterns among college students were associated with delayed circadian cycles and lower academic performance. (Philips et all, 2017)

\section{Conclusion:-}

When the scores of memory test had been categorized, the differences between cases and controls became significant. But there is not significant association between time sleeping and high memory strength; however, there is significant association between time of sleeping and low memory strength. Furthermore, there is no effect of level of education and income on memory strength; whilst sex and marital status has effect on memory of night people (i.e. cases); while age group has effect on memory of control group.

\section{Acknowledgments:-}

I would like to present my gratitude to every one helped me to collect, review and check the data. I'm thankful for all participants. Special thanks to my sister Aisheh, my brother Dr. Abdullah; my nieces Heba, Noor, Malakk; my nephews Amjed and Akram. Also, lots of thanks to Miss Arwa AlAjlouni ; Miss Badeaa Naasan and her coworkers; Rasha AlTahat, to my sister-in-law Nabeela, Fatmah, Suhailah and my cousin Feryal Akour.

\section{Conflicts Of Interest}

The author declares no conflict of interest. Financial disclosure: none. 


\section{References:-}

1. Christoph R. (2009 Dec) "Proactive People Are Morning People.” Journal of Applied Social Psychology 39, no. 12: 2787-2797.

2. Doghramji K. (2006). The Epidemiology and Diagnosis of Insomnia. Am J Manag Care. 12:S214-S220.

3. DOS. (2016). Jordan statistical yearbook. Department of Statistics, Amman: Jordan.

4. El Hangouche A. J., Jniene A., Aboudrar S., Errguig L., Rkain H., Cherti M., \& Dakka T. (2018). Relationship between poor quality sleep, excessive daytime sleepiness and low academic performance in medical students. Adv Med Educ Pract. 9: 631-638.

5. Faith Xue. (2019). Scientific studies say this is the best time to sleep. Byrdie, Dotdash publishing family: New York.

6. Giri P., Baviskar M., \& Phalke D. (2013). Study of sleep habits and sleep problems among medical students of pravara institute of medical sciences loni, Western maharashtra, India. Annals of medical and health sciences research, 3(1), 51-54.

7. Gupta S.S. (2015). Why is it not considered good to sleep at sunset? Retrieved April 29, 2019, From https://www.quora.com/Why-is-it-not-considered-good-to-sleep-at-sunset.

8. Hirshkowitz M. et al. (2015). National Sleep Foundation's sleep time duration recommendations: methodology and results summary. Sleep Health 1, 40-43.

9. Jean-Louis G., von Gizycki H., Zizi F., Nunes J. (1998 Oct). Mood states and sleepiness in college students: influences of age, sex, habitual sleep, and substance use. Percept Mot Skills. 87(2):507-12.

10. Jordan Times. (2018 Sep). 'Illiteracy rate in Jordan stood at 5.2 per cent in 2017'. Jordan: Amman. Retrieved from http://www.jordantimes.com/news/local/illiteracy- rate-jordan-stood-52-cent-2017, 2019)

11. Kohyama J. (2007). Early rising children are more active than late risers. Neuropsychiatric disease and treatment, 3(6), 959-963.

12. Lauderdale D. S., Knutson K. L., Yan L. L., Liu K., \& Rathouz P. J. (2008). Self- reported and measured sleep duration: how similar are they?. Epidemiology (Cambridge, Mass.), 19(6), 838-845.

13. Mishra A., Pandey R. K., Minz A., and Arora V. (2017 Dec). Sleeping habits among school children and their effects on sleep pattern. J Caring Sci. 6(4): 315-323.

14. Mograss M.A., Guillem F., Stickgold R. (2010). Individual Differences in Face Recognition Memory: Comparison among Habitual Short, Average, and Long Sleepers . Behav Brain Res. 208(2): 576-583. doi:10.1016/j.bbr.2010.01.002.

15. Mograss M. A. (2008). The influence of sleep on memory . Ph.D. dissertation. McGill University, Canada.

16. Morselli L. et al. (2010). Role of sleep duration in the regulation of glucose metabolism and appetite. Best Pract Res Clin Endocrinol Metab 24(5):687-702.

17. Phillips A.J., Clerx. W.M., O’Brien C., Sano A., Barger L.K., Picard R.W., ... Czeisler C. A. (2017). "Irregular sleep/wake patterns are associated with poorer academic performance and delayed circadian and sleep/wake timing." Scientific Reports vo R. lume 7, Article number: 3216.

18. Potkin K. T., \& Bunney W. E. Jr (2012). Sleep improves memory: the effect of sleep on long term memory in early adolescence. PloS one, 7(8), e42191.

19. Scott S.J. (2018). Do smart and rich people stay awake at night and sleep until noon? Oldtown Publishing LLC., NJ.

20. Smith M., Robinson L., \& Segal R. (2018). Your Guide to Healthy Sleep. The National Institutes of Health.

21. SSRF. (2012). Sleeping late at night - how it affects us? Spiritual Science Research Foundation Inc. Mumbai, India.

22. Taylor Jones. (2017 May). How many hours of sleep do you really need? Healthline newsletter. San Francisco and New York

23. UN. (2017). United nations demographic yearbook. United Nations, New York: USA.

24. Walton A.G. (2015 Nov). The sleep habits of highly successful people. Forbes Media LLC, New York. 